\title{
Improving the documentation of nasogastric tube insertion and adherence to local enteral nutrition guidelines
}

\author{
Esther Cole
}

Noble's Hospital, Isle of Man

\begin{abstract}
Fine bore nasogastric (NG) tubes are often required for patients who have insufficient nutrition to meet their daily requirements, as well as for feeding or medications when there are difficulties with swallowing. "Death or severe harm as a result of a naso [...] gastric tubes being misplaced in the respiratory tract" is one of the Department of Health's list of "never events". Noble's Hospital, Isle of Man, has local guidelines based on the National Patient Safety Agency's 2005 guidelines and 2011 update, regarding the initial insertion and confirmation of placement of NG tubes.
\end{abstract}

Retrospective baseline data looking at 13 case notes across 10 hospital wards showed that the majority of NG tube insertions took place on the stroke unit. A three-point quality of guidelines score showed that $8 / 13(62 \%)$ cases were following guidelines appropriately. A seven-point quality of documentation score showed no case notes had full documentation.

A teaching intervention for junior doctors and nurses was devised. However, there was no significant improvement in quality scores after 90 days (49 NG tube insertions). Therefore, an NG tube bundle, which included a pro forma for the case notes, information poster, and sticker for the nurse notes, was trialled on the stroke unit for six weeks. This showed that $10 / 12(83 \%)$ cases were following guidelines appropriately. While only $2 / 12(16 \%)$ of case notes had full documentation, this represented the two occasions when the pro forma was filled in and filed correctly. It is hoped that there could be a roll out of the intervention hospital-wide with identification of ways to improve usage of the NG tube bundle.

\section{Problem}

Fine bore nasogastric (NG) tubes are frequently required for patients who have insufficient nutrition to meet their daily requirements, or for feeding or medications when there are swallowing difficulties.

Appropriate insertion and the checking of correct placement of $\mathrm{NG}$ tubes is essential to avoid harm to patients. "Death or severe harm as a result of a naso- [...] gastric tubes being misplaced in the respiratory tract" is one of the Department of Health's list of "never events", a largely preventable patient safety incident that should not occur if the available preventative measures have been implemented.

Recent clinical incidents involving NG tubes at Nobles Hospital, Isle of Man, have anecdotally affected staff confidence and caused a change in attitude towards NG tubes, with possible deviation from the local and national guidelines. As such, it was felt that a quality improvement project to address these concerns would be desirable.

\section{Background}

The National Patient Safety Agency (NPSA) has issued guidance to NHS organisations in England and Wales, relating to adults, children and infants, entitled "Reducing the harm caused by misplaced nasogastric feeding tubes". This was followed in 2011 by an update in response to further clinical incidents. In 2005, the NPSA stated that 11 deaths and one case of serious harm due to misplaced nasogastric (NG) tubes had occurred over a two-year period. The 2011 NPSA update revealed that the NPSA's National Reporting and Learning System (NRLS) had received reports of a further 21 deaths and 79 cases of harm since September 2005. Noble's Hospital is the main hospital on the Isle of Man, a crown dependency distinct from the UK; it has local guidelines based on the above NPSA guidelines.

The NPSA 2005 guidelines state that for standard NG tube insertions in adult patients, the first-line method of checking the placement of NG tubes should be by obtaining an aspirate of between $\mathrm{pH} 1$ and 5.5 . This reliably shows the NG tube to be in the stomach, rather than the lung. If this is unsuccessful, the secondline method of checking placement is to perform a chest $x$ ray. Additional methods such as "the whoosh test" and the colour of aspirate and litmus paper should be avoided.

For high risk NG tube insertions, such as unconscious patients in ITU or for patients with swallowing difficulties or absent gag reflex, these should be confirmed by chest $x$ ray in the first instance, though this decision depends on clinical judgement. The NPSA 2011 update reinforced the above guidelines, while also setting out a specific set of steps each time an NG tube is inserted, to ensure the following were considered:

a) Is NG feeding the right decision for this patient? 
BMJ Quality Improvement Reports

b) Is this the right time to place the NG tube and is the appropriate equipment available?

c) Is there sufficient knowledge/expertise available at this time to test for safe placement of the NG tube?

In response to further clinical incidents where chest $x$ rays were wrongly interpreted, it was also suggested that:

i) $X$ ray request forms clearly state that the purpose of the $x$ ray is to establish the position of the NG tube for the purpose of feeding

ii) The radiographer takes responsibility to ensure that the NG tube can be clearly seen on the $\mathrm{x}$ ray to be used to confirm tube position

iii) Documentation of the tube placement checking process includes confirmation that any $\mathrm{x}$ ray viewed was the most current $\mathrm{x}$ ray for the correct patient, how placement was interpreted, and clear instructions as to required actions. Any tubes identified to be in the lung are removed immediately, whether in the $\mathrm{x}$ ray department or clinical area.

The above guidelines have a broad scope. As such, it was realised during the course of the quality improvement project that it should have a specifically focus, ie initial insertion of NG tubes and confirmation of placement, using the NPSA 2005 guidelines as the agreed standard. We were aiming for $100 \%$ attainment. A time frame of 90 days was initially proposed, however, this was later extended to 120 days as the project developed.

\section{Baseline measurement}

For this project, three baseline measures were proposed. The first was the process measure of how many NG tubes were being inserted. The second was an outcome measure of how many of these NG tubes had been inserted in accordance with the local guidelines. The third was a process measure to see whether documentation in patient case notes was adequate.

It was decided not to use an outcome measurement (such as morbidity or mortality) as the sample size would be small and these are rare occurrences.

Prospective data collection was to start on 11th November 2013. Therefore, it was decided to retrospectively collect data on NG tubes placed in the preceding four weeks for use as baseline data. These patients were identified by asking staff on 10 hospital wards if they remembered any patients who had recently had an NG tube inserted. As such, there may be an element of selection bias. What this did identify was that there was no formal system of measurement in place at that time to identify patients who were having NG tubes inserted.

Using this method, $13 \mathrm{NG}$ tube insertions were reviewed. Eight of these NG tubes were inserted on ward 7, the stoke unit.

Subsequent data confirmed that the majority of NG tube insertions take place here. A quality score out of 5 was devised to assess whether these NG tube insertions were done in accordance with local guidelines, as follows:

1. There is a clear and appropriate indication for NG tube insertion

2. It is the right time to place the NG tube and there is the appropriate equipment available

3. There is sufficient knowledge/expertise available at this time to test for safe placement of the NG tube?

4. The NG tube position is confirmed appropriately:

- The first-line method of checking NG tube placement is by aspirate of 5.5 or less

- The second-line method of checking NG tube placement is by chest $x$ ray

1. 5. No inappropriate tests were used, eg litmus paper/x ray as first-line (except in high risk patients)/whoosh test, etc.

However, after the second PDSA cycle it became apparent that points 2 and 3 (based on the NPSA 2011 update) were too subjective. They were therefore removed from the analysis and all data retrospectively analyzed using a simplified three-point quality score. This meant that data collected was more objective and took less time to be collected and analyzed.

A seven-point quality of documentation score to record a minimum data-set was also devised, as follows:

1. There is documentation of a decision/indication for NG tube

2. There is documentation of the NG tube insertion

3. There is documentation of the length of NG tube insertion

4. There is documentation of which nostril the NG tube was inserted into

5. There is documentation of whether an aspirate was obtained or not

6. There is documentation of whether NG tube placement has been checked and what method was used - either an aspirate with a $\mathrm{pH} 5.5$ or less, or a chest $\mathrm{x}$ ray which is documented in the case notes that it is the most recent and the four anatomical criteria to confirm placement

7. There were instructions written as to required actions, eg safe to feed, needs to be pushed in further etc.

The three-point quality of guidelines score showed that 8/13 (62\%) cases were following guidelines appropriately. The seven-point quality of documentation score showed no case notes had full documentation. However, it did highlight a marked discrepancy between quality of documentation score when looking at documentation in case notes (average score of 2/7 (29\%)) compared to nurse and case notes combined (average Score of $4 / 7$ $(57 \%))$

See supplementary file: ds4027.xlsx - "Baseline Data"

\section{Design}

As NG tube insertion is principally a procedure that falls to nurses 
and junior doctors, it was decided that an intervention should be aimed at these two groups. Different options for interventions were considered, including a pro forma to improve documentation, or a change to the way chest $x$ rays were reported. The level of knowledge of staff was felt to be a crucial factor affecting an anecdotal lack of confidence and this would indirectly improve documentation in case notes, as well as ensuring guidelines were followed. The intervention could be adapted based on gaps in the knowledge identified from a baseline questionnaire.

A teaching session was therefore devised to be included as part of the foundation teaching programme for F1 and F2 doctors, to cover the indications, contraindications, and complications of NG tube insertion, as well as the local and national guidelines. Though briefly touched upon, interpretation of chest $x$ rays was felt to be beyond the scope of a single session as trainees were at different stages of their training. Therefore a link was included to provide further training via the e-learning module recommended by the NPSA (www.trainingngt.co.uk).

A teaching session for junior doctors was felt to be sustainable for the following reasons: i. if effective, teaching on NG tubes could be included in the induction of new doctors to the Trust or have a permanent place in the foundation teaching programme ii. Teaching would be accessible, as it would be attended by the majority of foundation year 1 and 2 doctors iii. It would be cost-effective, as there were no initial cost required, but the benefits of reducing inappropriate investigations and reducing patient harm would save money iv. The intervention was patient-centric, addressing an important patient safety issue.

The proposal for the teaching session was taken to the director of medical education and medical education manager, who agreed for the session to be included in the foundation teaching programme, scheduled for December 2013.

Targeting the education of nursing staff would be more difficult as there was not the same opportunity to encounter all nurses at the same time. A teaching intervention was devised whereby nurses on each ward would be offered education in rotation. This would be more time-consuming, but would offer similar benefits to those outlined above.

\section{Strategy}

PDSA cycle 1: Education sessions were devised targeting foundation doctors and ward nurses. The foundation year 1 and 2 doctors were given a session as part of their teaching programme. This covered the indications for NG tube insertion, the national guidelines regarding checking placement, and principles of good documentation. Meanwhile, a teaching programme for the nursing staff was rolled out ward by ward over several weeks.

Unfortunately, nursing attendance was poor. There was no improvement in quality scores after a 90 day period (49 NG tube insertions across medical, surgical and critical care wards).

PDSA cycle 2: A different sort of intervention was therefore devised. This took the form of an NG tube bundle for use when an NG tube was inserted. It consisted of a pro forma (that would be filed in the case notes), a sticker for the nursing notes (to indicate that a pro forma was in use), and an information poster (with the local NG tube guidelines). To address some of the limitations in the early part of the project, data collection was simplified by rolling out the intervention only on ward 7 , the stroke unit. This is where the majority of NG tubes are inserted. The data showed that NG tube guidelines were, on the whole, being followed. But the pro forma was not being filled in appropriately or correctly filed; therefore, documentation scores remained poor in the case notes, though good in the nursing notes.

PDSA cycle 3 to 6: Several PDSA cycles were done in quick succession over six weeks to make small alterations to the pro forma encourage correct usage. However, other factors were starting to be identified that were limiting the success of the pro forma, such as the separate use of nursing notes and case notes for documentation by nurses and doctors respectively, a lack of awareness of the quality improvement project etc. These factors would need to be taken into account when rolling out the pro forma across the hospital.

See supplementary file: ds5326.docx - "Outline of PDSA Cycles"

\section{Post-measurement}

Baseline data of $13 \mathrm{NG}$ tube insertions suggested that there were marked discrepancies between quality of documentation in case notes compared to nursing notes and case notes combined, and that adherence to the guidelines was variable. The first PDSA cycle took 90 days and showed that over $49 \mathrm{NG}$ tube insertions across the hospital the teaching intervention had made no real difference to quality scores for adherence to guidelines. Similarly, there was no obvious improvement in quality of documentation in case note scores.

An NG tube bundle was therefore introduced to focus on improving quality of documentation scores in case notes. This took place over six weeks and involved small numbers, only 12 insertions in total, but showed that on the three occasions when the pro forma was filled in correctly and filed correctly, quality scores for documentation in the case notes could increase from low scores of 0 or 1 , up to high scores of 6 or 7 .

See supplementary file: ds5323.xlsx - "Run charts and data tables"

\section{Lessons and limitations}

At the outset of the project, it was envisaged that it would be completed within 90 days. However, for a number of reasons the first PDSA cycle was cumbersome and laborious.

Firstly, it was felt that too much data was collected. An initial quality score for adherence to guidelines was a five-point score, including two subjective points that were time-consuming to ascertain and difficult to standardise. Once the data was re-analysed with these two points removed, it was much easier to analyse the data in a 
meaningful way and move forward.

Secondly, the quality improvement project was logistically challenging as data was being collected over 10 wards across the whole hospital. It was therefore necessary to devise a way of finding out whether NG tubes had been inserted across the whole hospital and go between wards collecting data. This involved liaising with dieticians to be pick up new referrals for enteral feeding and telephoning the wards at intervals to see if NG tubes had been reinserted. Once it was decided to trial a new intervention on a single ward, this made both data collection and analysis easier so that PDSA cycles could go ahead more quickly.

Thirdly, it was felt that too much was trying to be achieved in the first PDSA cycle, ie to improve both adherence to guidelines and quality of documentation. To overcome this, subsequent PDSA cycles focused mainly on the area of documentation in case notes. This again helped to speed up PDSA cycles and allowed lots of small adjustments to the intervention, rather than trying to get it right first time.

After the first PDSA cycle and the challenges faced, it was difficult to know how to move forward. Advice was therefore sought from various mentors regarding how to creatively overcome difficulties. This included the Royal College of Physicians 'Learning to Make a Difference' programme, and engagement with individuals locally who were aware of quality improvement methodology. This highlighted the value of having individuals within an organisation who can advise regarding projects. There is currently no quality improvement lead at Noble's Hospital, but this is something that has been highlighted as being potentially beneficial.

There were other local factors that needed to be addressed before the project could move forward. These may have also stemmed from a lack of awareness of quality improvement methodology at Noble's Hospital, which could be overcome by having a quality improvement lead.

Firstly, there were difficulties seeking "audit approval" from the local audit department. Unfortunately, as the audit department were unfamiliar with the concept of quality improvement projects as distinct from clinical audit, this meant that audit approval had to be obtained under the guise of a "re-audit", as ultimately several audit cycles would be performed.

Secondly, once an intervention involving documentation was devised, it was necessary to obtain documentation committee approval. There was some confusion as to how to go about this and whether or not it was necessary. As such, it took time for the correct person to be contacted. The difficulties obtaining approval have been fed back locally and a review of this committee is currently under way.

Finally, one of the potential strengths of the quality improvement project was the involvement of individuals from multiple disciplines. This allowed the successful trial of an intervention on the stroke unit. In retrospect, it would have been beneficial to have this multidisciplinary approach earlier in the project. As such, the initial PDSA cycle could have run more efficiently with earlier identification that the teaching intervention was not having the desired impact. In the latter stages of the project, this approach meant that there was regular feedback from stakeholders and help with overcoming challenges as they arose.

\section{Conclusion}

Often it is the successful quality improvement projects that are presented or published. This project encountered a number of logistic, methodological and local challenges that needed to be overcome to get to the point finally reached ie an intervention trialled on a small scale, in a single setting, that showed some potential to improve clinical practice, rather than outright success.

However, it will hopefully have highlighted some local systemsbased limitations that can now be addressed to ensure future quality improvement projects can avoid some of the challenges faced. Similarly, it has given weight to the argument that a local quality improvement lead may be beneficial to set in motion and support future quality improvement projects, as well as increasing local awareness of quality improvement methodology.

Similarly, the challenges faced in the initial stages of the project and how to overcome them may be of value to others embarking on quality improvement projects. There is a temptation to collect large amounts of data without a clear focus, rather than small amounts of useful focused data; to trial interventions on a large scale, rather than testing on a small scale without the expectation of getting it right first time; the value of a multi-disciplinary approach was only realised in the latter stages of the project, but in retrospect could have been in place from the outset.

Finally, the sustainability and usefulness of the intervention is unclear. While there was potential for documentation scores to improve when the intervention was successfully implemented, the sample size was small and impact of the intervention inconsistent. A number of other factors were identified that would limit the successful roll out of the intervention across the rest of the hospital which would need to be addressed.

\section{References}

1. National Patient Safety Agency. Reducing the harm caused by misplaced nasogastric feeding tubes - Patient Safety Alert (2005)

http://www.nrls.npsa.nhs.uk/EasySiteWeb/getresource.axd? Asset $\mid \mathrm{D}=60002 \&$ type $=$ full $\&$ servicetype $=$ Attachment (accessed 11 Nov 2013).

2. National Patient Safety Agency. NPSA - Reducing the harm caused by misplaced nasogastric feeding tubes in adults, children and infants (2011) http://www.nrls.npsa.nhs.uk/EasySiteWeb/getresource.axd? Asset $\mid \mathrm{D}=129696 \&$ type $=$ full $\&$ servicetype $=$ Attachment (accessed 11 Nov 2013).

3. Department of Health. The 'Never Events' List 2012-13 (2012) 


\section{BMJ Quality Improvement Reports}

https://www.gov.uk/government/uploads/system/uploads/att achment_data/file/215206/dh_132352.pdf (accessed 11 Nov 2013)

\section{Declaration of interests}

Nothing to declare.

\section{Acknowledgements}

Dr John Thomas (lead consultant), Jackie White (PEG nurse specialist), Rachel Cleator (dietician), Gill Horsey (stroke nurse specialist), Elaine Quayle (senior nurse, stroke unit), Kate Mcgowan (senior nurse, stroke unit).

\section{Ethical approval}

According to the policy activities that constitute research at the Noble's Hospital, Isle of Man, this work met criteria for operational improvement activities exempt from ethics review. 